\title{
Fraction vegetation cover extraction of winter wheat based on RGB image obtained by UAV
}

\author{
Huanbo Yang ${ }^{1,2}$, Jing Zhao ${ }^{1,2^{*}}$, Yubin Lan ${ }^{1,2}$, Liqun $\mathrm{Lu}^{2,3}$, Zhiming $\mathrm{Li}^{1,2}$ \\ (1. School of Agricultural and Food Engineering, Shandong University of Technology, Zibo 255000, China; \\ 2. International Precision Agriculture Aviation Application Technology Research Center, Shandong University of Technology, \\ Zibo, 255000, China; \\ 3. School of Transportation and Vehicle Engineering, Shandong University of Technology, Zibo, 255000, China)
}

\begin{abstract}
In order to quickly and accurately extract the fraction vegetation cover (FVC) of winter wheat, the unmanned aerial vehicle (UAV) was used to obtain the visible light image of winter wheat during the green returned stage, and the estimation ability of winter wheat FVC based on visible light vegetation index and texture feature was explored, 8 texture features of winter wheat images were extracted by gray-level co-occurrence matrix, and 3 visible light vegetation indices of VDVI, NGBDI and GRVI were calculated. According to the characteristics of vegetation and soil blue \& green and green \& red bands in the research field, the green-red combined vegetation index (GRDI) and green-blue combined vegetation index (GBVI) were constructed. In addition, regression models of vegetation cover were established using 5 vegetation indices and 8 texture features respectively. The results showed that the regression model established by GRDI had the highest accuracy among vegetation indices with $R^{2}$ value of 0.9245 , RMSE value of 0.02677 , and nRMSE value of $5.74 \%$. Therefore, the GRDI model was selected in this paper to generate FVC level distribution map, which provided a basis for winter wheat growth monitoring and field management.
\end{abstract}

Keywords: winter wheat, FVC, UAV remote sensing technology, texture feature, vegetation index construction DOI: $10.33440 /$ j.ijpaa.20190202.44.

Citation: Yang H B, Zhao J, Lan Y B, Lu L Q, Li Z M. Fraction vegetation cover extraction of winter wheat based on spectral information and texture features obtained by UAV. Int J Precis Agric Aviat, 2019; 2(2): 54-61.

\section{Introduction}

Wheat green returned stage is a key period to promote the growth of late weak seedlings, control the length of the seedlings, adjust the size of the yield and determine the rate of spike formation $^{[1]}$. Fraction vegetation cover is defined as the vertical projection of the crown or shoot area of vegetation canopies from the ground surface, and is expressed as a fraction or percent of the reference area ${ }^{[2-4]}$. The FVC of winter wheat during the green returned stage is monitored reasonably, and the prescription map of FVC level distribution is generated, which are of great significance for guiding the topdressing and irrigation of winter wheat during the green returned stage. FVC extraction is mainly achieved by satellite remote sensing and ground remote sensing ${ }^{[5-7]}$. The data of satellite remote sensing has the disadvantages of low spatial, temporal resolution, vulnerability to atmospheric influences and high cost, and it is often difficult to effectively meet the requirements of FVC extraction at the field scale ${ }^{[8,9]}$. Remote

Received date: 2019-11-28 Accepted date: 2019-12-15

Biographies: Huanbo Yang, Postgraduate student, research interests: Application of UAV Remote Sensing Technology in Agricultural, Email: 1332616134@qq.com; Yubin Lan, PhD, research interest: precision agricultural aviation application, Email: ylan@scau.edu.cn; Liqun Lu, PhD, research interests: Special vehicle design, fluid transmission and control technology, Email: ylan@scau.edu.cn; Zhiming Li, Postgraduate student, research interests: Application of UAV Remote Sensing Technology in Agricultural Monitoring Direction, Email: 2295386984@qq.com;

*Corresponding author: Jing Zhao, PhD, research interests: agricultural remote sensing technology and intelligent detection. School of Agricultural and Food Engineering, Shandong University of Technology, Zibo City, Shandong, 255000, China. Email: zbceozj@163.com. sensing images on the ground can effectively monitor the FVC of crop, however, it is difficult to be applied to the extraction of crop vegetation coverage in large areas due to the limitations of human and material condition ${ }^{[9]}$. In a word UAV remote sensing technology has been widely used because of its unique flexibility, high image resolution and low cost.

Previous studies on FVC monitoring have been carried out $^{[10-19]}$ by remote sensing technology. The common FVC extraction is mostly based on the vegetation index threshold method, linear spectral mixture mode, machine learning and regression model. It was difficult for vegetation index threshold method to determine the classification threshold of vegetation and soil effectively. The linear spectral mixture mode was greatly affected by the resolution of remote sensing images in the process of extracting $\mathrm{FVC}$, the endmember of low resolution remote sensing images are often difficult to extract accurately. The machine learning method had high requirements for sample selection and computer performance when extracted FVC from large plots. The extraction of FVC based on regression model is easy to implement, which required low image resolution and computer performance.

The FVC extraction model was mainly established by vegetation indices and texture features. Most vegetation indices were constructed based on visible and near-infrared bands, such as $\mathrm{NDVI}^{[20,21]}$, SAVI ${ }^{[22]}$, and ARVI ${ }^{[23]}$, but most UAV on the market were only equipped with visible light digital cameras. Therefore, the vegetation indices based on the near-infrared band was difficult to apply. Normalized green-blue difference index (NGBDI) ${ }^{[24]}$, visible band difference vegetation index (VDVI) ${ }^{[11]}$ and green-red vegetation index (GRVI) ${ }^{[25]}$ have been constructed by predecessors according to the spectral reflectance characteristics of green 
vegetation in the visible light band, However, FVC is low in areas where crops do not flourish, and the soil background has a great impact on the common visible vegetation index. The real-time extraction and analysis of the index vegetation information can easily result in big errors ${ }^{[15]}$.

At present, the extraction of FVC is mainly achieved through multispectral imagery ${ }^{[5,12]}$, there are few reports on the use of UAV visible light remote sensing to provide a basis for field management of winter wheat during the green returned stage. In order to study the method of rapid FVC extraction and provide a basis for efficient and accurate field management of winter wheat, it was analyzed that spectral features and texture features of winter wheat extracted from visible light images obtained by UAV.

\section{Materials and Methods}

\subsection{Research field}

The experiment was carried out on a field in Huantai county located in the north of Zibo city, China (36 $\left.58^{\prime} 34^{\prime \prime} \mathrm{N}, 118^{\circ} 3^{\prime} 44^{\prime \prime} \mathrm{E}\right)$. Zibo City is a national temperate monsoon climate arear of warm temperate zone, the average annual precipitation is $650 \mathrm{~mm}$ and the average annual temperature is $12.5^{\circ} \mathrm{C}-14.2^{\circ} \mathrm{C}$. Winter wheat was planted by machine in December 2018, and the data of winter wheat was acquired during the green returned stage (March 14, 2019). 9 reference plates were arranged in the test area for geometric correction. The research field was approximately $2520 \mathrm{~m}^{2}$. An overview of the research field is shown in Figure 1.

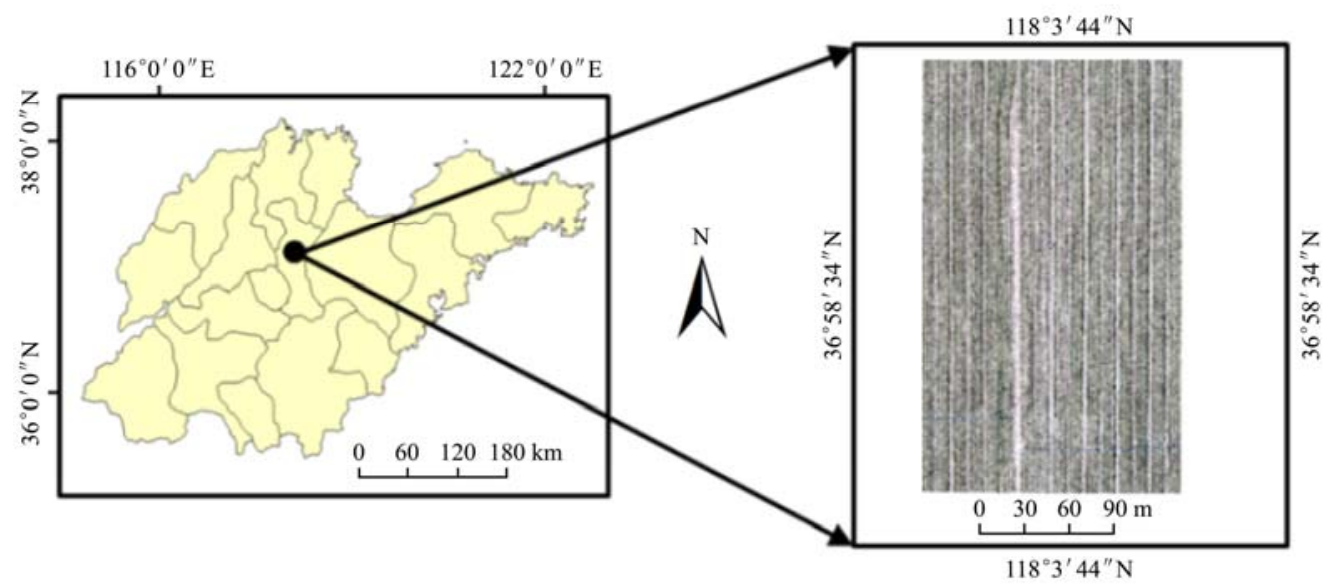

Figure 1 Research field overviews

\subsection{Acquisition and preprocess of UAV visible light image}

The experiment data collected by the UAV visible light remote sensing image was carried out at $1 \mathrm{~cm}$ resolution. During this experiment, DJI Phantom 4 Advanced (Shenzhen Dajiang Baiwang Technology Co., Ltd., Shenzhen, China) was adopted to collect visible light image of winter wheat during the green returned stage, which has a remote sensing platform with a CMOS digital camera to perform a continuous flight over the $60000 \mathrm{~m}^{2}$ studying area. The cameral had an $84^{\circ}$ field of view lens with an $f / 2.8$ aperture and a resolution of $5472 \times 3648$ pixels. The digital camera was de-noised, and lens distortion correction was performed before using. Its main parameters were shown in Table 1. In order to reduce the influence of light conditions on the acquisition of remote sensing data by UAV, data acquisition was performed at around $12 \mathrm{pm}$. in sunny and windless weather. According to specific weather condition, and white balance was set to sunny. Flight was controlled by Altizure software (Everest Innovation Technology Ltd., Hong Kong, China), which directed the UAV flying along a serpentine image acquisition plan at a height of $40 \mathrm{~m}$ and a speed of $4 \mathrm{~m} / \mathrm{s}$ with the camera looking downwards. Overlap of image to the front and side was $80 \%$, and 245 remote sensing images were acquired. To improve the geo-location accuracy, the geo-referencing of the point clouds was done using a combination of direct geo-referencing and 9 ground control points.

After acquisition, the image mosaic processing was performed by using Pix4DMapper software (Pix4D Inc., Switzerland, https://www.pix4d.com/) which was specifically designed to process UAV image using techniques rooted in both computer vision and photogrammetry ${ }^{[26]}$. The processing workflow was: initializing all geo-located images captured in each flight by automatically searching for feature tie-points in match pairs of images and correcting them based on the camera model. After that, nine ground control points were incorporated to correct the geographic coordinates of image. Finally, the digital surface model and orthophoto was generated by using the inverse distance weighting method. More detailed information about the image mosaic processing based on Pix4DMapper software can be seen in $^{[27,28]}$.

Table 1 Main parameters of UAV visible light image acquisition system

\begin{tabular}{lc}
\hline \multicolumn{1}{c}{ Parameter } & value \\
\hline Weight & $1368 \mathrm{~g}$ \\
Flight time & $30 \mathrm{~min}$ \\
Speed & $<72 \mathrm{~km} / \mathrm{h}$ \\
Image sensor & 1 -inch CMOS \\
Lens focal length & $8.8 \mathrm{~mm} / 24 \mathrm{~mm}$ \\
ISO range & $100-12800$ \\
Shutter speed & $8-1 / 8000 \mathrm{~s}$ \\
\hline
\end{tabular}

In order to extract the FVC of winter wheat, the estimation model of FVC based on vegetation indices and texture features were established during the green returned stage. The main steps included: 1) orthophoto of research field spliced by Pix4DMapper software was classified into wheat and soil based on support Vector Machine (SVM), and the confusion matrix was used to verify the classification results which were used as true value for the establishment and evaluation of FVC estimation model. 2) The common visible light vegetation indices were calculated, and two new vegetation indices were constructed by analyzing the spectral characteristics of soil and wheat vegetation during the green returned stage. The texture features of the image were extracted by gray-level co-occurrence matrix, and the correlation between texture features and FVC was analyzed. Regression model were 
performed on texture features with high correlation coefficients. 3) The FVC estimation models based on vegetation indices and texture features were established and verified. 4) The regression model with higher accuracy was selected to generate the FVC level distribution map of winter wheat during the green returned stage. The flow chart was shown in Figure 2.

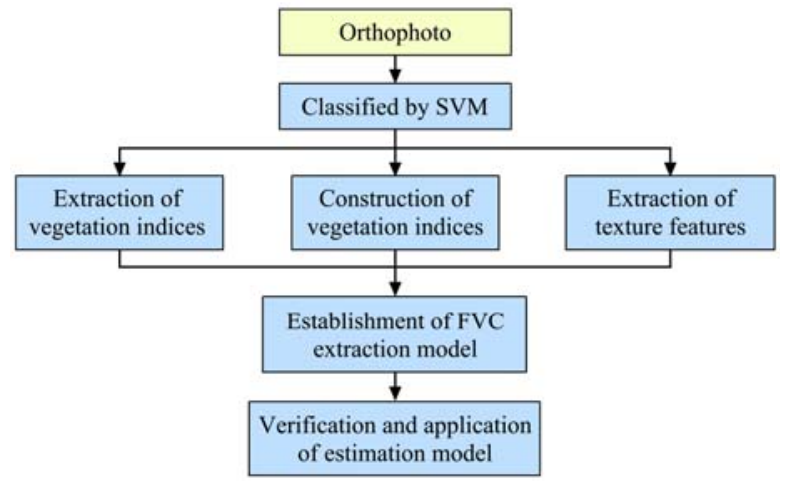

Figure 2 Schematic of main procedures to extraction of winter wheat FVC

2.3 Classification of research field image and verification of classification result

In this experiment, the classification results of the winter wheat based on SVM during the green returned stage were used as the true value. SVM was an emerging statistical machine classification method proposed by Vapnic with good application in recent years ${ }^{[29]}$, which could effectively solve problems such as small sample, nonlinearity, high dimension, and has strong generalization ability.

The confusion matrix was the method of verifying classification results proposed by Congalton in 1991, which could not only calculate the indicators representing the overall classification accuracy, such as total accuracy, kappa coefficient, but also calculate the indicators representing the classification of individual category such as producer accuracy and user accuracy. Moreover, the confusion matrix could be used as the input of advanced statistical techniques. Therefore, confusion matrix method was proposed as the standard method for verifying results accuracy of remote sensing classification. This paper uses the confusion matrix method to verify the accuracy of the SVM classification result of winter wheat.

\subsection{Calculation of UAV visible light vegetation indices}

Three vegetation indices (VDVI, NGBDI and GRVI) were selected to establish FVC regression models of winter wheat during the green returned stage. The calculation formulas of vegetation indices were shown in (1)-(3).

$$
\begin{gathered}
V D V I=\frac{2 G-R-B}{2 G+R+B} \\
N G B D I=\frac{G-B}{G+B} \\
G R D I=\frac{G-R}{G+R}
\end{gathered}
$$

$\mathrm{G}, \mathrm{R}$ and $\mathrm{B}$ represent greyscale values of green, red and blue images respectively.

\subsection{Construction of GRDI and GBVI}

The visible light vegetation indices were constructed by analyzing the spectral characteristics of wheat and soil, and comparing the differences of greyscale values in red, green and blue images, which were finally used to generate the FVC level distribution map of winter wheat during the green returned stage.
60 wheat and soil region of interest (ROI) were selected on the visible light image of winter wheat. The eigenvalues (including maximum, minimum, average and standard deviation) of the pixels in the red, green and blue bands of wheat and soil samples were counted. It was found that the greyscale values of the above images were crossed and could not be using a single band to accurately extract FVC information of wheat. According to the above problem, scatter plots of the combination of red, green and blue greyscale values of wheat and soil were displayed. The scatter plots of the combination of red \&green and green \& blue greyscale values of wheat and soil were selected based on the apparent dividing line between wheat and soil scatter plots (Figure 3). As shown in Figure 3, there were two clear boundaries between wheat and soil scatter plots based on red \& green and blue \& green axes. Wheat is basically distributed at the upper left of the boundary line, and the soil is basically distributed at the lower right of the boundary. The dividing line scatter coordinate value was extracted by visual interpretation method, 53 points were read on the two scatter plots for fitting, respectively, then the expression of feature combination parameters could be obtained. The fitting results were shown in Figure 4. The fitted boundary function expression are taken as the newly constructed vegetation index GBVI and GRDI, and the GBVI and GRDI formulas are shown in (4) (5), and the greyscale images of GBVI and GRDI are shown in Figure 5.

$$
\begin{gathered}
G R D I=G-1.1282 R+7.2613 \\
G B V I=G-1.0545 B+4.903
\end{gathered}
$$
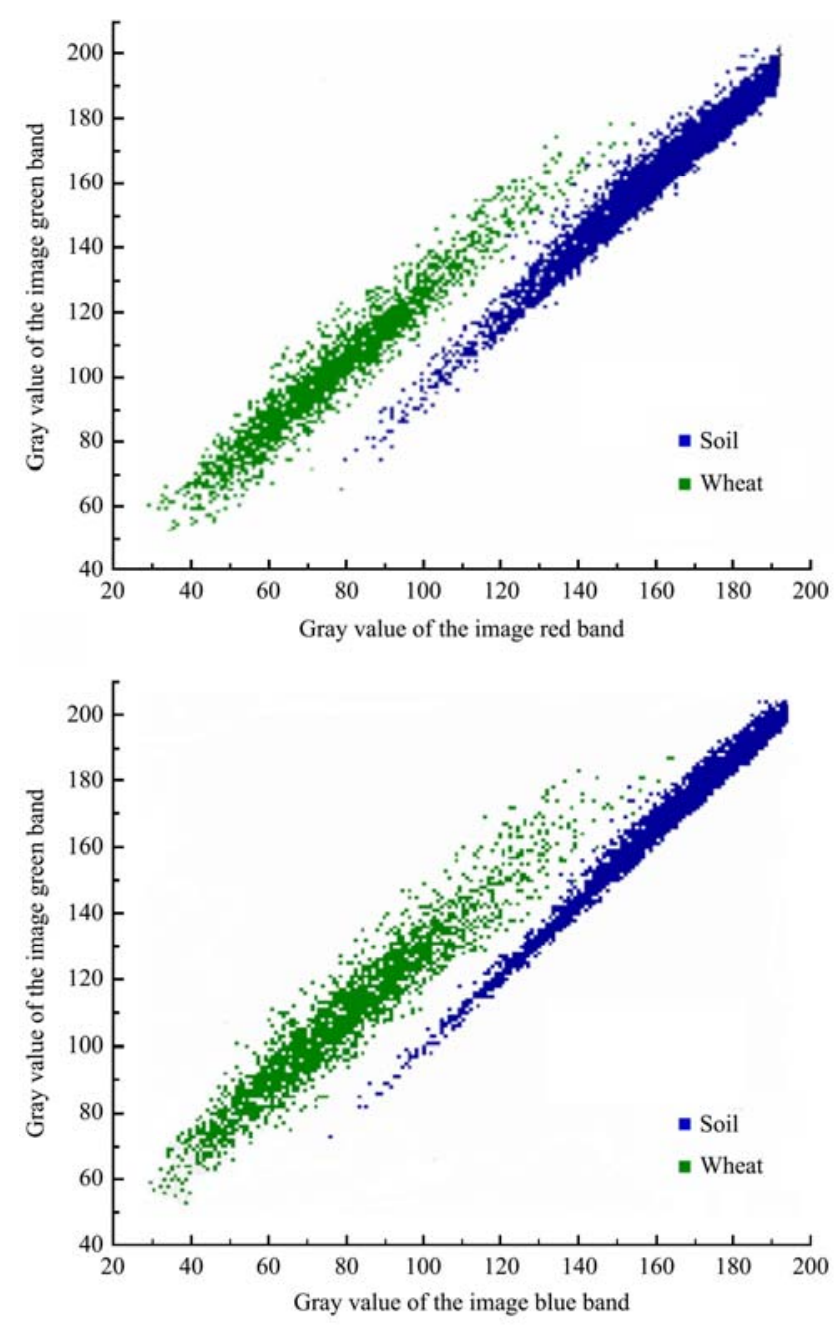

Figure 3 Scatter plots of wheat and soil 

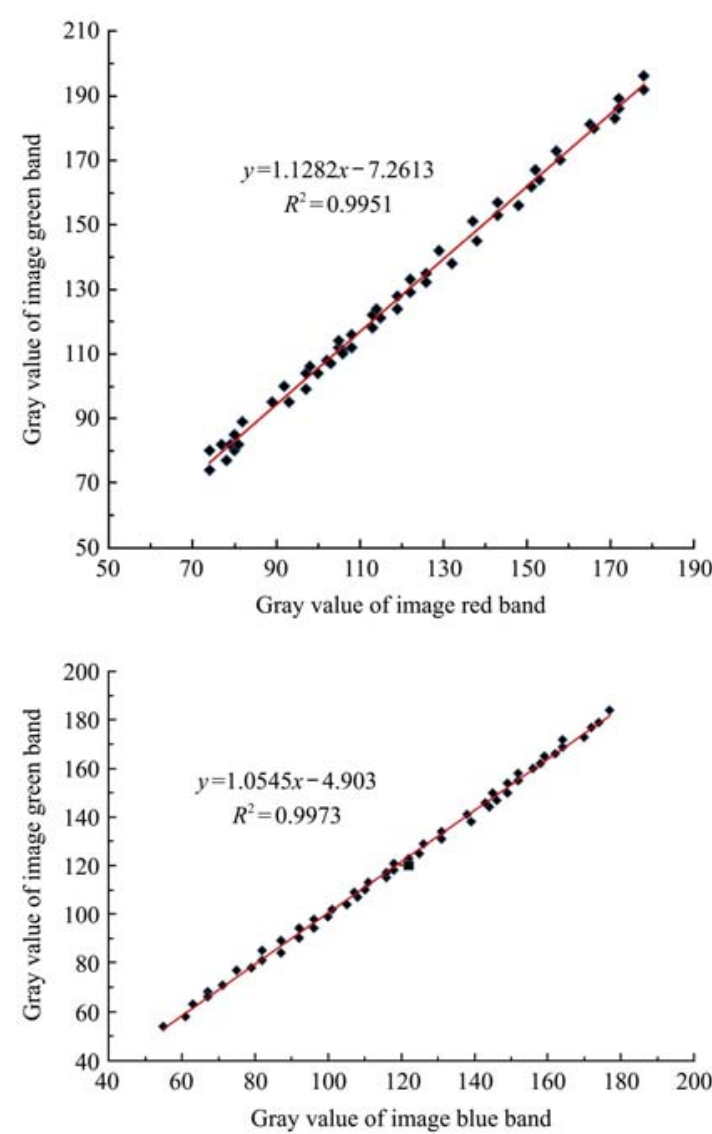

Figure 4 Fitting of VBVI and VRDI

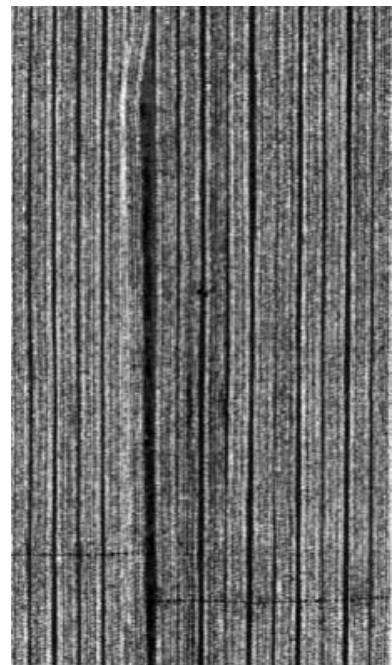

GRDI

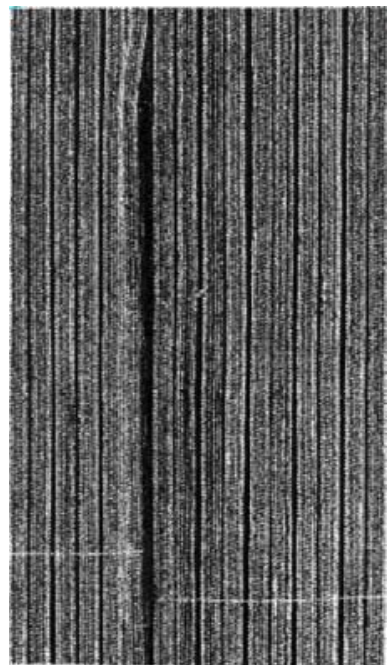

GBVI
Figure 5 Greyscale image of GBVI and GRDI

\subsection{Extraction and selection of texture features}

The gray-level co-occurrence matrix is a method for extracting image texture features with calculating the conditional probability density function between greyscale levels of images ${ }^{[29]}$.

In this paper, the Mean value, Variance, Homogeneity, Contrast, Dissimilarity, Entropy, Angular Second Moment and Correlation were extracted from windows of $3 \times 3,5 \times 5,7 \times 7,9 \times 9$, $11 \times 11,13 \times 13$ and $15 \times 15$. Arcmap software was used to obtain the mean value of the texture features of each band in the research field image, and a total of 168 texture features were acquired.

SPSS 22.0 software (IBM, USA) was used to analyze the correlation between the texture features and the FVC of winter wheat by pearson correlation coefficient method, and then the texture features of high correlation were adopted to set up the FVC estimation model.

\subsection{Methods of modeling and validation}

46 groups of sampling areas were selected as the modeling set and 36 groups of sampling areas as the verification set on the orthophoto of winter wheat. The vegetation index and texture features were used to establish the FVC regression model of winter wheat. The regression models between vegetation indices and FVC were established with unitary linear regression. Considering that there may be multicollinearity problem in the texture features of the orthophoto of winter wheat, partial least squares (PLS) method was selected to established estimation model between texture features and FVC. The PLS method integrated the advantages of principal component analysis, canonical correlation analysis and linear regression, and could effectively solve the problems of multicollinearity and few examples ${ }^{[30,31]}$. The accuracy of the model was evaluated by using three factors: coefficients of determination $\left(R^{2}\right)$, root mean square error (RMSE) and normalized root mean square error (nRMSE), the closer $R^{2}$ was to 1 , the smaller the RMSE and nRMSE were, the better the model was. The formulas of $R^{2}$, RMSE and nRMSE are shown in (6)-(8).

$$
\begin{gathered}
R^{2}=\frac{\sum_{i=1}^{n}\left(X_{i}-\bar{X}\right)^{2}\left(Y_{i}-\bar{Y}\right)^{2}}{\sum_{i=1}^{n}\left(X_{i}-\bar{X}\right)^{2} \sum_{i=1}^{n}\left(Y_{i}-\bar{Y}\right)^{2}} \\
R M S E=\sqrt{\frac{\sum_{i=1}^{n}\left(Y_{i}-X_{i}\right)^{2}}{n}} \\
n R M S E=\frac{R M S E}{\bar{X}} \times 100 \%
\end{gathered}
$$

where, $X_{i}, \bar{X}$ respectively represent the true value of the FVC and the average of FVC true value; $Y_{i}, \bar{Y}$ respectively represent the estimated value of the FVC and the average of FVC estimated values.

\section{Results and discussion}

\subsection{SVM classification and results analysis}

It was used as training sets for classification with SVM that 60 wheat and soil samples were selected during the construction of the above vegetation indices. The results showed that the separability between samples was 1.996. Generally, the value of two parameters was between 0 and 2, and the value was greater than 1.9, indicating that the separability between samples was good and qualified. Using the same sample selection criteria, 50 wheat and soil ROI from another region were selected as validation sets. The classification accuracy of the SVM classification results was verified by the confusion matrix. The results showed that the overall classification accuracy was $98.9773 \%$, and the Kappa coefficient was 0.9793 . The soil and wheat classification results are shown in Table 2. It can be seen from Table 2 that the classification results accuracy of SVM is calculated.

Table 2 Results of confusion matrix verification

\begin{tabular}{ccccc}
\hline Class name & $\begin{array}{c}\text { Soil } \\
\text { /pixels }\end{array}$ & $\begin{array}{c}\text { Wheat } \\
\text { /pixels }\end{array}$ & $\begin{array}{c}\text { Total number of } \\
\text { samples/pixels }\end{array}$ & $\begin{array}{c}\text { User accuracy } \\
\text { /\% }\end{array}$ \\
\hline Soil & 11743 & 191 & 11934 & 98.40 \\
Wheat & 26 & 9259 & 89447 & 99.72 \\
$\begin{array}{c}\text { Total number of } \\
\text { samples/pixels }\end{array}$ & 11769 & 9450 & 201480 & \\
Producer accuracy/\% & 99.78 & 97.98 & & \\
\hline
\end{tabular}




\subsection{Establishment of regression model of FVC based on vegetation index}

The FVC information of 46 modeling datasets was counted in Arcmap, and it was used as the true value. The regression model was established with the 5 vegetation indices, and the 36 verification sets were used to verify the accuracy of the above regression model. The relationship between vegetation indices and FVC of winter wheat in the research field are shown in Figure 6.

It can be seen from Figure 6 that the correlation between vegetation index and FVC was extremely significant $(P<0.01)$, and the most relevant to the FVC of winter wheat during the green returned stage was GRDI, with a correlation coefficient of 0.9245 .
Followed by VDVI, GBVI, GRVI and NGBDI, the correlation coefficients were $0.9064,0.9026,0.5624$ and 0.4588 , respectively.

The 36 verification sets were used to validate the estimation model of winter wheat FVC, and FVC estimation model $\left(R^{2}\right.$, RMSE and nRMSE) were calculated (Figure 7). According to the verification results $\mathrm{l}$, the FVC regression model based on GRDI vegetation index showed the best results $\left(R^{2}=0.9231\right.$, RMSE $=$ 0.02011 , nRMSE $=5.74 \%)$, followed by VDVI $\left(R^{2}=0.913\right.$, RMSE $=$ 0.02634, nRMSE $=7.52 \%)$ and GBVI $\left(R^{2}=0.9078\right.$, RMSE $=0.0247$, nRMSE=7.06\%). In conclusion, GRDI and GBVI have better effect on inversion of FVC, which can effectively complete the FVC monitoring of winter wheat during the green returned stage.
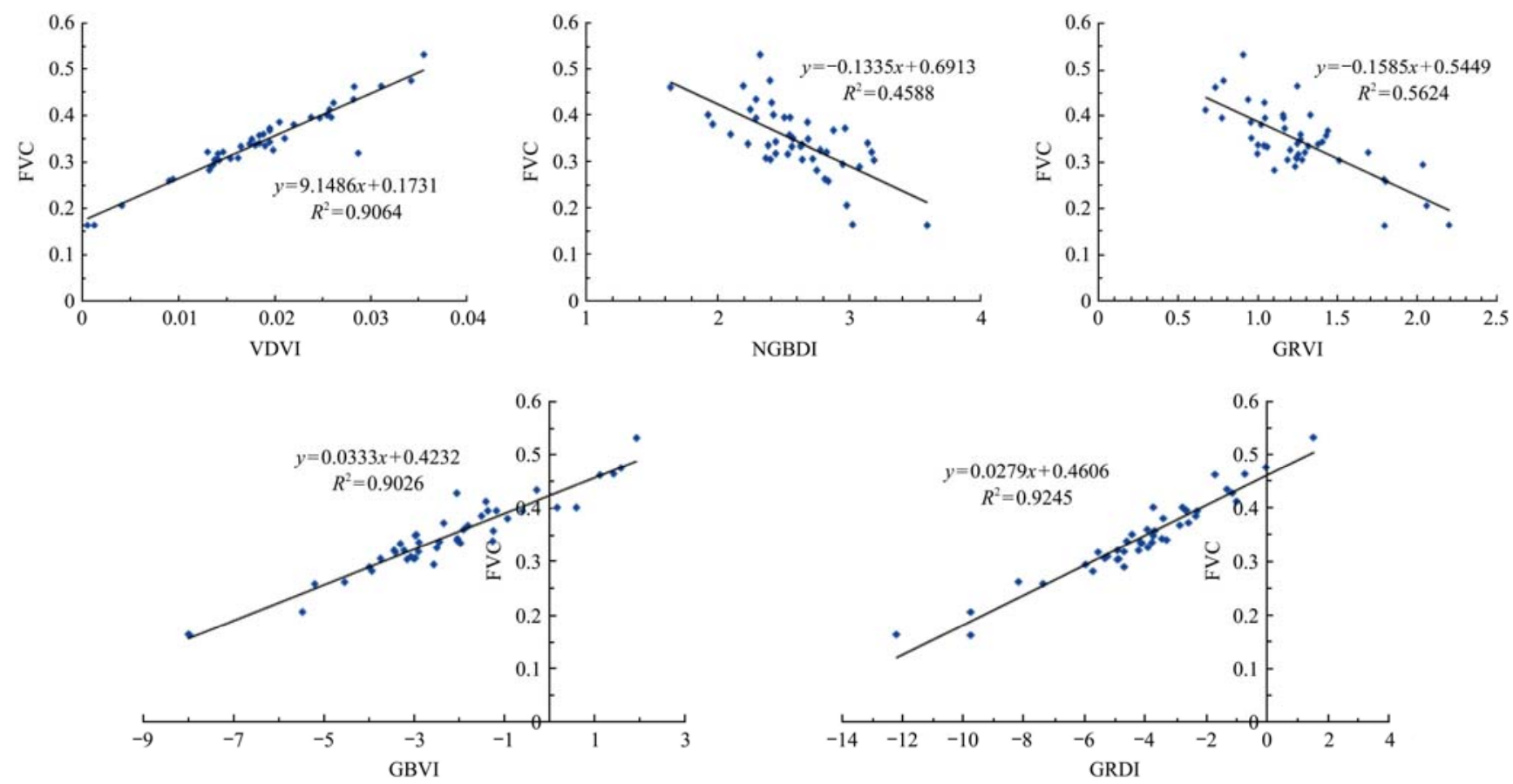

Figure 6 Relationship between vegetation indices and FVC

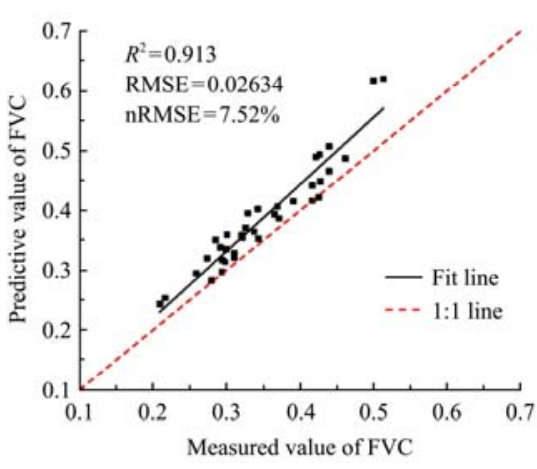

a. VDVI

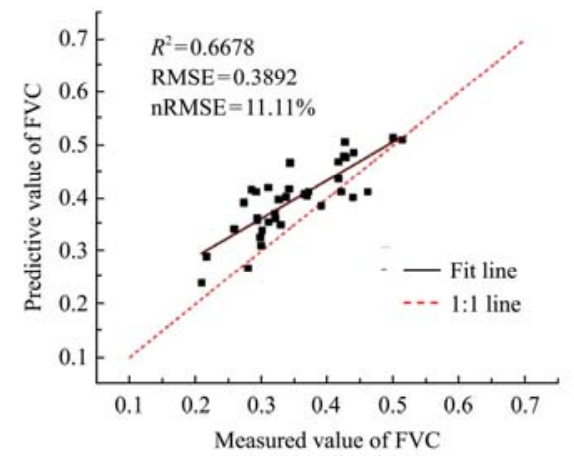

b. NGBDI

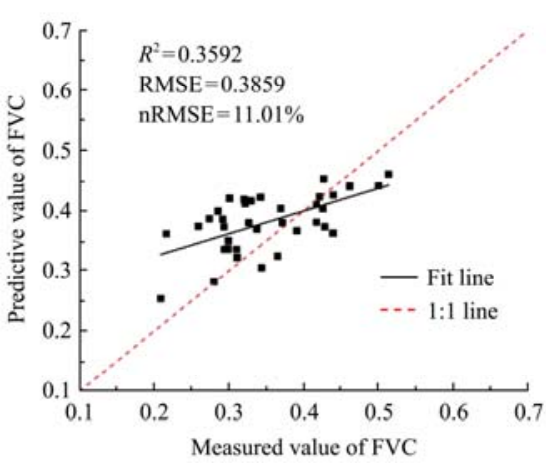

c. GRVI

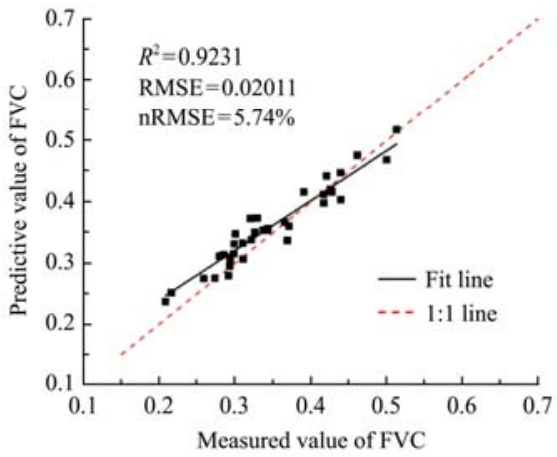

d. GRDI

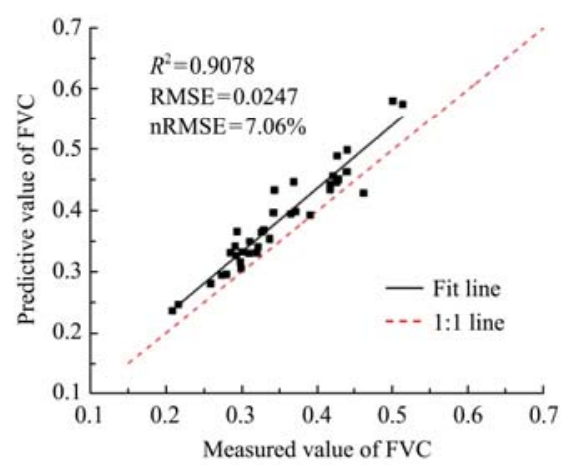

e. GBVI

Figure 7 Relationship between measured values and predicted values of FVC based on vegetation indices 
However, the inversion results of NGBDI and GRVI were not ideal. By analyzing the greyscale images of NGBDI and GRVI, it was found that the greyscale values difference of NGBDI and GRVI between soil and wheat were not obvious. The greyscale images of NGBDI and GRVI are shown in Figure 8.

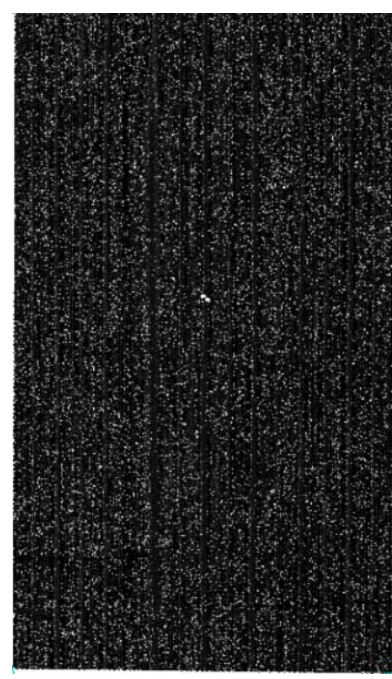

NGBDI

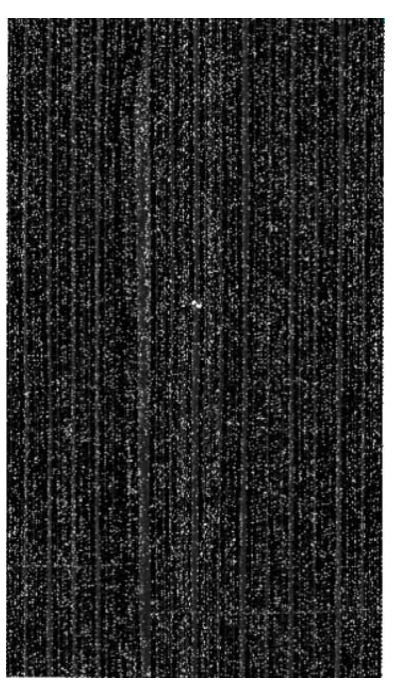

GRVI
Figure 8 Greyscale image of NGBVI and GRVI

\subsection{Analysis on texture features of winter wheat}

The average of 8 texture features (R, G and B bands) under 7 windows in 46 groups of sampling areas were calculate by Arcmap software, and it was used as a modeling factor to conduct correlation analysis with the truth values of FVC during the green returned stage of winter wheat. The mean value of the red band $\left(R^{2}=0.893, P<0.01\right)$ under the $5 \times 5$ window, the mean value of the blue band $\left(R^{2}=0.889, P<0.01\right)$ under the $7 \times 7$ window and the mean value of the green band $\left(R^{2}=0.819, P<0.01\right)$ under the $13 \times 13$ window were selected. The analysis of correlation coefficient matrix was performed on three kinds of texture features selected above, and the results were shown in Table 3. It can be seen from the correlation coefficient matrix that $R^{2}$ of the three texture features were greater than 0.95 , which proved that the above texture features have strong correlation. To further illustrate the matter of collinearity, the variance inflation factor (VIF) was used to characterize its severity (Table 4). It is generally recommended that when a VIF of independent variable is greater than 10 , it indicates that there is strong collinearity between the independent variables. It can be seen from Table 4 that the variance inflation factors of the three texture features were all greater than 10 , further indicating that the three texture features had severe collinearity.

Table 3 Correlation coefficient matrix of wheat texture feature

\begin{tabular}{cccc}
\hline Band type & $\begin{array}{c}\text { Mean value of the } \\
\text { blue band }\end{array}$ & $\begin{array}{c}\text { Mean value of the } \\
\text { green band }\end{array}$ & $\begin{array}{c}\text { Mean value of the } \\
\text { red band }\end{array}$ \\
\hline $\begin{array}{c}\text { Mean value of the } \\
\text { blue band }\end{array}$ & 1 & 0.99 & 0.98 \\
$\begin{array}{c}\text { Mean value of the } \\
\text { green band } \\
\begin{array}{c}\text { Mean value of the } \\
\text { red band }\end{array}\end{array}$ & 0.98 & 1 & 0.98 \\
\hline
\end{tabular}

Table 4 VIF statistics of wheat texture feature

\begin{tabular}{lcc}
\hline \multicolumn{1}{c}{ Band type } & Tolerance & VIF \\
\hline Mean value of the blue band & 0.012 & 82.09 \\
Mean value of the green band & 0.012 & 85.765 \\
Mean value of the red band & 0.024 & 41.342 \\
\hline
\end{tabular}

\subsection{Establishment of regression model of FVC based on} texture features

Due to the serious collinearity problem of texture features, the regression model was established by the traditional least square method, which will lead to large errors in the accuracy of the model with small changes in the independent variables. The collinearity problem of the above texture features can be effectively solved by PLS method. PLS regression model of wheat FVC was established by three texture features selected above $\left(R^{2}=0.9507\right.$, $F=169.47, P<0.01)$. The regression equation is shown in (9).

$$
Y=-0.074 X_{1}+0.132 X_{2}-0.068 X_{3}+0.564
$$

where, $X_{1}, X_{2}, X_{3}$ respectively represent the mean value of red band under the $5 \times 5$ window, the mean value of blue band under the $7 \times 7$ window, and the mean value of green band under the $13 \times 13$ window.

The regression model established by PLS method was validated using 36 verification sets. The results of the verification were shown in Figure $9\left(R^{2}=0.9507\right.$, RMSE $=0.0167$, nRMSE $=$ $4.76 \%)$. It can be seen from the verification results that the regression model of FVC with the above three texture features based on PLS method had higher accuracy.

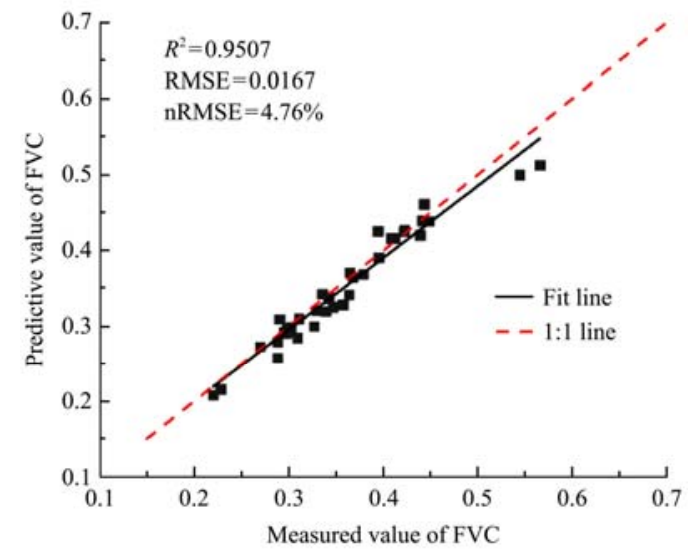

Figure 9 Relationship between measured values and predicted values of FVC based on texture feature extraction

\subsection{Application of FVC extraction model}

The regression model based on GRDI vegetation index and the model based on texture feature were selected with the highest accuracy of winter wheat FVC extraction. When the FVC of winter wheat in the research field was extracted and mapped, it was found that the model based on texture features easily identified non-vegetation pixels as vegetation. The error recognition results are shown in Figure 10, where the green part represents wheat vegetation and the white part represents the soil. The main reason for the misidentification of PLS regression model was that there were other materials such as straw in the research field. The regression model established by the mean value of $R, G$ and $B$ bands had certain limitations for the extraction FVC of farmland in complex environments.

Considering misidentification of FVC extraction model based on texture features during the green returned stage, the regression model established by GRDI vegetation index was used to map FVC level distribution during the green returned stage of winter wheat (Figure 10). Figure 10 showed that the FVC extraction results of winter wheat during the green returned stage were divided into three levels, I represents the low coverage area, II represents the medium coverage area, and III represents the high coverage area. The number of pixels and the proportion of each level were counted. The statistical results are shown in Table 5. 


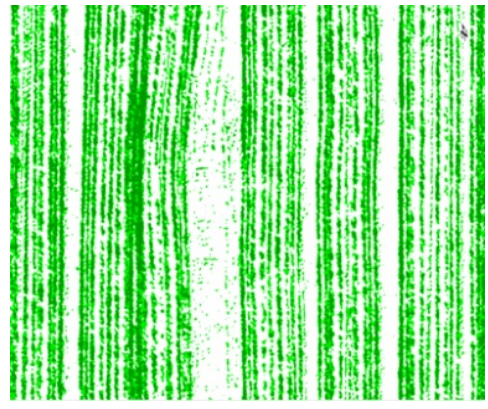

a. Mapping results of texture features

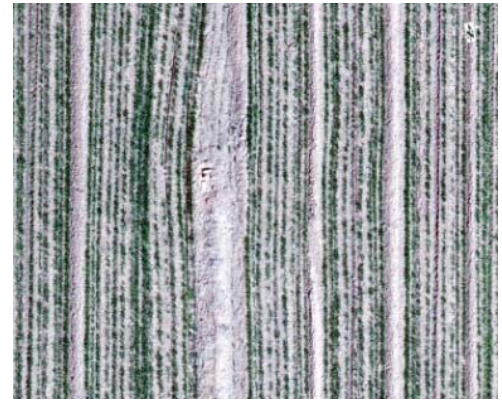

b. Original visible light image

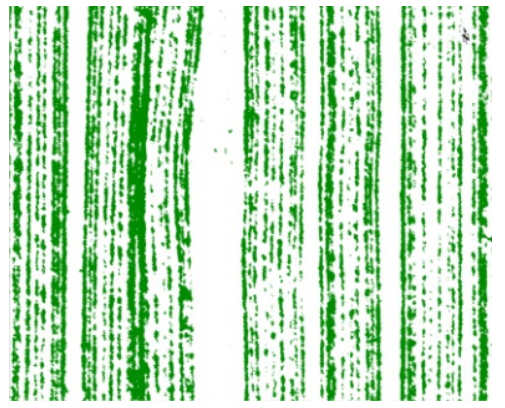

c. Classified image by SVM

Figure 10 The problem of FVC misidentification based on texture features model

According to the statistical results of FVC in winter wheat, the low FVC area in the research field had a higher proportion, followed by the medium coverage area and the high coverage area. At present, it is rarely reported that study about the level on FVC distribution of winter wheat based on the empirical model with UAV visible light images during the green returned stage The distribution map of winter wheat FVC level not only provides a basis for the management of winter wheat field, but also provides a theoretical basis for subsequent related research.

Table 5 Statistical analysis of FVC based on GRDI vegetation index

\begin{tabular}{cccc}
\hline Level & FVC & Number of pixels & The proportion/\% \\
\hline I & $0-0.3$ & 13058421 & 56.2130 \\
II & $0.3-0.6$ & 5135444 & 22.1095 \\
III & $0.6-1$ & 5033494 & 21.6705 \\
\hline
\end{tabular}

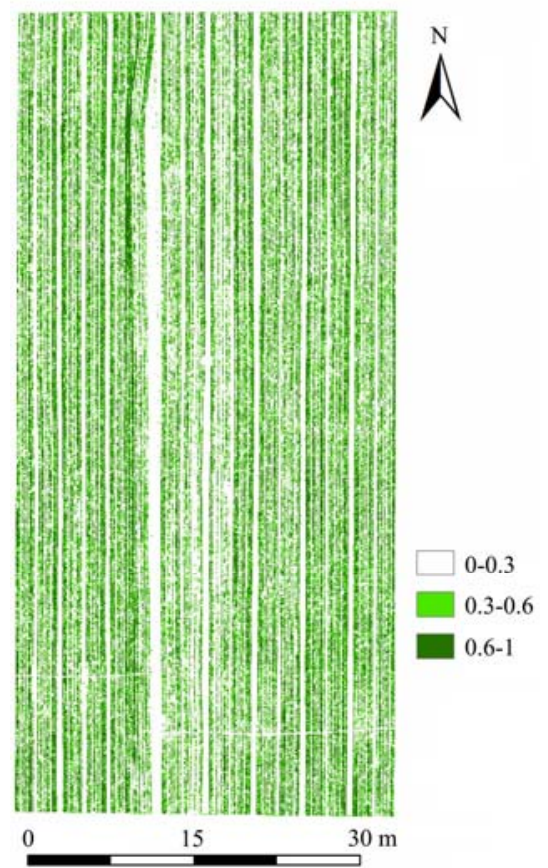

Figure 11 FVC level distribution of wheat based on GRDI vegetation index

\section{Conclusion}

1) The vegetation indices of GBVI and GRVI were constructed by analyzing the spectral information (R, G and $B$ images) of winter wheat and soil, which could effectively extract the FVC information of winter wheat during the green returned stage.

2) It has higher correlation with the FVC that mean values (R, $\mathrm{G}$ and $\mathrm{B}$ images) extracted by the gray-level co-occurrence matrix. When the estimation model established with the texture features is used to extract the FVC of winter wheat during the green returned stage, it is easy to identify the non-vegetation pixels as vegetation.

3) Compared with the FVC regression model established by GBVI, VDVI, NGBDI and NGRDI, the FVC of GRVI is best with R2 value of 0.9231, RMSE value of 0.02011 and nRMSE value of $5.74 \%$.

\section{Acknowledgements}

The authors gratefully acknowledge the financial support provided by Top Talents Program for One Case One Discussion of Shandong Province, The Development Special Funds on Science and Technology to Guide Local by the Central Government "Research and Development on Technology and Equipment of Precision Agriculture Aviation", Science and Technology Development Program of Zibo (2018kj010073), Program of Shandong Provincial Collaborative Innovation Center of Dry-farming Intelligent Agricultural Equipment.

\section{[References]}

[1] Liu J H, Zhang W F. Study on the application of wheat population rapid diagnosis method for mobile phone-end during the green returned stage. Agriculture and Technology, 2018, 38(06): 1-4. doi: 10.11974/ nyyjs.20180333001. (in Chinese)

[2] Gu Z, Ju W, Li L, et al. Using vegetation indices and texture measures to estimate vegetation fractional coverage (VFC) of planted and natural forests in Nanjing city, China. Advances in Space Research, 2013, 51(7): 1186-1194. doi: 10.1016/j.asr.2012.11.015.

[3] Zhang D, Mansaray L R, Jin H, et al. A universal estimation model of fractional FVC for different crops based on time series digital photographs. Computers \& Electronics in Agriculture, 2018, 151: 93-103. doi: 10.1016/j.compag.2018.05.030

[4] PUREVDOR J TS, TATEISHI R, ISHIYAMA T, et al. Relationships between percent FVC and vegetation indices. International Journal of Remote Sensing, 1998, 19(18): 3519-3535. doi: 10.1080/ 014311698213795.

[5] Niu Y X, Zhang L Y, Han W T, et al. Winter wheat coverage extraction method based on UAV remote sensing and vegetation index. Transactions of the Chinese Society of Agricultural Machinery, 2018, 49(4): 212-221. doi: 10.6041/j.issn.1000-1298.2018.04.024. (in Chinese)

[6] Zhang D D, Mansaray L R, Jin H W, et al. A universal estimation model of fractional vegetation cover for different crops based on time series digital photographs. Computers and Electronics in Agriculture, 2018, 151: 93-103. doi: 10.1016/j.compag.2018.05.030.

[7] Feng Q L, Liu J T, Gong J H. Urban Flood Mapping Based on Unmanned Aerial Vehicle Remote Sensing and Random Forest Classifier-A Case of Yuyao, China. Water, 2015, 7(12): 1437-1455. doi: 10.3390/w7041437.

[8] Zhang X, Zhang F, Qi Y, et al. New research methods for vegetation information extraction based on visible light remote sensing images from an unmanned aerial vehicle (UAV). International Journal of Applied Earth Observation and Geoinformation, 2019, 78: 215-226. doi: 10.1016/ j.jag.2019.01.001

[9] Zhao J, Yang H B, Lan Y B, Lu L Q, Jia P, Li Z M. Extraction Method of Summer Corn FVC Based on Visible Light Image of Unmanned Aerial 
Vehicle. Transactions of the Chinese Society for Agricultural Machinery, 2019, 50(5): 232-240. doi: 10. 6041 j.issn.1000-1298.2019.05.027. (in Chinese)

[10] Zhang D, Mansaray L R, Jin H, et al. A universal estimation model of fractional FVC for different crops based on time series digital photographs. Computers \& Electronics in Agriculture, 2018, 151: 93-103. doi: 10.1016/j.compag.2018.05.030.

[11] Wang X Q, Wang M M, Wang S Q, et al. Extraction of vegetation information from visible unmanned aerial vehicle images. Transactions of CSAE, 2015, 31(5): 152-158. doi: 10.3969/j.issn.1002-6819.2015.05.022 (in Chinese)

[12] Li B, Liu R Y, Liu S H, et al. Monitoring FVC variation of winter wheat by low- altitude UAV remote sensing system. Transactions of the CSAE, 2012, 28(13): 160-165. doi: 10.3969/j.issn.1002-6819.2012.13.026. (in Chinese)

[13] Sun Z P, Liu S H, Jiang J, et al. Coordination inversion methods for FVC of winter wheat by multi-source satellite images. Transactions of the CSAE, 2017, 33(16): 161-167. doi: 10.11975/j.issn.1002-6819.2017.16.021 (in Chinese)

[14] Chen H B, Huang B B, Peng D L. Comparison of pixel decomposition models for the estimation of fractional FVC. Journal of Northwest Forestry University, 2018, 33(03): 203-207. doi: 10.3969/ j.issn1001-7461.2018.03.31 ( in Chinese)

[15] Bian X, Ma Q Y, Liu C Y. FVC Calculation Based on Low Altitude Visible Spectrum. Bulletin of Soil and Water Conservation, 2017, 37(5): 270-275. doi: 10.13961/j.cnki.stbctb.2017.05.046. (in Chinese)

[16] Xihan M, Wanjuan S, Zhan G, et al. Fractional vegetation cover estimation by using multi-angle vegetation index. Remote Sensing of Environment, 2018, 216: 44-56. doi: 10.1016/j.rse.2018.06.022.

[17] H Godínez-Alvarez, Herrick J E, Mattocks M, et al. Comparison of three vegetation monitoring methods: Their relative utility for ecological assessment and monitoring. Ecological Indicators, 2009, 9(5): 0-1008. doi: 10.1016/j.ecolind.2008.11.011

[18] Jiapaer G, Chen X, Bao A. A comparison of methods for estimating fractional FVC in arid regions. Agricultural and Forest Meteorology, 2011, 151(12): 0-1710. doi: 10.1016/j.agrformet.2011.07.004

[19] Niu Y X, Zhang L Y, Han W T. Extraction Methods of Cotton Coverage Based on Lab Color Space. Transactions of the Chinese Society of Agricultural Machinery, 2018, 49(10): 240-249. doi: 10.6041/ j. issn.1000-1298.2018.10.027 (in Chinese)

[20] Rouse Jr., J.W., Haas, R., Schell, J., Deering, D., 1973. Monitoring
Vegetation Systems in the Great Plains With ERTS. Third ERTS Symposium, NASA SP-351. NASA, Washington, DC, pp. 309-317.

[21] Tucker, C.J., 1979. Red and photographic infrared linear combinations for monitoring vegetation. Remote Sens. Environ. 8, 127-150. doi: 10.1016/0034-4257(79)90013-0

[22] Huete A. A soil-adjusted vegetation index (SAVI). Remote Sensing of Environment, 1988, 25(3): 295-309. doi: 10.1016/0034-4257(88)90106-X.

[23] Kaufman, Y. J, Tanre, D. Atmospherically resistant vegetation index (ARVI) for EOS-MODIS. IEEE Transactions on Geoscience \& Remote Sensing, 1992, 30(2): 0-270. doi: 10.1109/36.134076.

[24] Hunt E R, Cavigelli M, Daughtry C S T, et al. Evaluation of Digital Photography from Model Aircraft for Remote Sensing of Crop Biomass and Nitrogen Status. Precision Agriculture, 2005, 6(4): 359-378. doi: 10.1109/36.134076.

[25] Takeshi M, Nishida N K, Hiroyuki O, et al. Applicability of Green-Red Vegetation Index for Remote Sensing of Vegetation Phenology. Remote Sensing, 2010, 2(10): 2369-2387. doi: 10.3390/rs2102369.

[26] Turner, D., Lucieer, A., Watson, C. An Automated Technique for Generating Georectified Mosaics from Ultra-High Resolution Unmanned Aerial Vehicle (UAV) Imagery, Based on Structure from Motion (SfM) Point Clouds. Remote Sens, 2012, 4, 1392-1410. doi: 10.3390/ rs4051392.

[27] Duan, T., Zheng, B., Guo, W., Ninomiya, S., Guo, Y., Chapman, S. C. Comparison of ground cover estimates from experiment plots in cotton, sorghum and sugarcane based on images and ortho-mosaics captured by UAV. Funct. Plant Biol, 2017, 44. doi: 10.1071/fp16123.

[28] Niu, Y., Zhang, L., Zhang, H., Han, W., Peng, X. Estimating Above-Ground Biomass of Maize Using Features Derived from UAV Based RGB Imagery. Remote Sens, 2019, 11, 1261. doi: 10.3390/ rs11111261.

[29] Vapnik, V. N. An overview of statistical learning theory. IEEE Transactions on Neural Networks, 1999, 10(5): 988-999. doi: 10.1109/ 72.788640 .

[30] Zhang Z T, Wang H F, Han W T, Bian J, Chen S B, Cui Ting. Inversion of Soil Moisture Content Based on Multispectral Remote Sensing of UAV. Transactions of the Chinese Society for Agricultural Machinery, 2018, 49(2): 173-181. doi: 10.6041 /j. issn.1000-1298.2019.07.027. (in Chinese)

[31] Yu L, Hong Y S, Geng L, et al. Hyperspectral estimation of soil organic matter content based on partial least squares regression. Transactions of the CSAE, 2015, 31(14): 103-109. doi: 10.11975/ j.issn.1002-6819.2015.14.015 (in Chinese) 